\title{
CA-125 AS A SURROGATE MARKER IN A CLINICAL AND HISTOPATHOLOGICAL STUDY OF PELVIC MASS AT A TERTIARY CARE HOSPITAL
}

Madhuri Kulkarni1 ${ }^{1}$ Ambarish Bhandiwad², Sunila R³, Sumangala4.

1. Professor, Department of Microbiology, JSS Medical College, Mysore.

2. Professor, Department of OBG, JSS Medical College, Mysore.

3. Professor, Department of Pathology, JSS Medical College, Mysore.

4. Senior Research Scholar, Department of OBG, JSS Medical College, Mysore.

\section{CORRESPONDING AUTHOR:}

Dr. Madhuri Kulkarni,

No 53, Ramashree,

$3^{\text {rd }}$ Cross, Dattagalli, E \& F Block,

RK Nagar, $1^{\text {st }}$ stage, Mysore-570022.

E-mail: mkmysore@gmail.com

\section{HOW TO CITE THIS ARTICLE:}

Madhuri Kulkarni, Ambarish Bhandiwad, Sunila R, Sumangala. "CA-125 as a Surrogate Marker in a Clinical and Histopathological Study of Pelvic Mass at a Tertiary Care Hospital". Journal of Evolution of Medical and Dental Sciences 2013; Vol2, Issue 26, July 1; Page: 4778-4782.

ABSTRACT: Context- Pelvic masses are common in women of all age groups and their accurate diagnosis poses a clinical challenge because of atypical and non specific presentation. The combination approach of exploratory laparotomy and histopathological examination remains the corner stone for management. CA 125, a biomarker has been evaluated in several studies as a surrogate tool in these cases. OBJECTIVES: To determine the diagnostic value of CA 125 in pelvic mass and to know its sensitivity and specificity in ovarian cancers. MATERIAL AND METHODS 80 patients suspected of pelvic mass at JSS Hospital, Mysore subjected to clinical examination, CA 125 detection and laparotomy followed by histopathological correlation. RESULTS: CA 125 levels were seen to be more in large fibroids. High levels were recorded in extensive adenomyosis and endometrial carcinoma stage $1 \mathrm{~A}$ onwards. Sensitivity and specificity in post-menopausal women was $100 \%$ for malignant ovarian tumors. CONCLUSION -CA 125 estimation can be used as an adjunct tool in the evaluation of pelvic mass and is a highly useful assay in the diagnosis of ovarian cancers in the post-menopausal women.

KEY WORDS: CA125, pelvic mass, ovarian cancers

INTRODUCTION: The finding of a pelvic mass usually causes great concern for the female patient and understandably such women want no stone unturned in the search for a diagnosis. The accurate diagnosis of pelvic mass poses a challenge to the attending physician because of its bizarre and atypical behavior. (1). Pelvic mass can range from adenomyosis, fibroid uterus, ovarian or fallopian tube cysts, ovarian or uterine malignancy or an inflammatory mass The management is also very varied and crucial; torsion of an ovarian cyst requires immediate surgery whereas an ovarian malignancy requires planned surgery and chemotherapy(2). Bridging the gap between the 
least invasive aid, i.e. pelvic examination and the invasive laparotomy is the biomarker CA125. It is a $200 \mathrm{KD}$ glycoprotein initially identified on the surface of the ovarian carcinoma cell line OVCA 433. (3). CA 125 is widely distributed on the surface of both healthy and malignant cells of mesothelial origin, including pleural, pericardial, peritoneal and endometrial cells, as well as in normal genital tract and amniotic membrane. Interestingly the molecule is not present on the surface of normal ovarian cells, but is present in $80 \%$ of malignant ovarian tissues of non-mucinous origin (4). The value of CA125 varies between laboratories depending on the type of assay used but levels less than $35 \mathrm{u} / \mathrm{ml}$ are considered to be normal (5).

In view of the wide distribution of CA125 expression, serum CA125 levels can be raised in various benign and inflammatory conditions. Also CA125 estimation has been used as a screening test in women with family history of ovarian cancer and for post operative detection of recurrence and monitoring response to therapy.(6).

This prospective study was undertaken to determine the diagnostic value of CA125 estimation in the evaluation of pelvic mass, by the departments of Obstetrics and Gynaecology, Microbiology and Pathology at JSS Hospital, Mysore.

MATERIAL AND METHODS: Eighty patients with provisional diagnosis of pelvic mass were enrolled in the study Known cases of choriocarcinoma and intra and extra uterine gestation were excluded. After detailed history taking, patients were subjected to thorough clinical examinations, baseline investigations and estimation of CA125 by ELISA. Exploratory laparotomy was done and specimens sent for histopathological examination. CA125 levels were correlated to the type of pelvic mass, determined by histopathology.

RESULTS: The majority of pelvic masses were seen in patients in the age group of 31-60 years (78.7\%), 57 patients (71.2\%) were pre menopausal and 23 (28.7\%) were post-menopausal. 28.7\% $(23 / 80)$ of the pelvic masses were more than 24 weeks size, 48 (60) were less than 24 weeks size and size could not be made out in 9 cases. Distribution of various associated conditions has been depicted in fig.1. Hypertension was the commonest co-morbidity, followed by diabetes mellitus. The frequency of different pelvic masses has been shown in table 2 and the type of ovarian tumours has been represented in table3. The correlation of clinical diagnosis with levels of CA125 has been denoted in table 4.

The following significant observations were made

1. The CA125 levels were more in larger fibroids of the seven clinically diagnosed cases of fibroid uterus, 3 showed CA125 levels more than $35 \mathrm{u} / \mathrm{ml}$ and 2 of these on histopathology revealed extensive adenomyosis.

2. Of the 4 cases of endometrial carcinoma, 2 had elevated CA125 levels and histopathology revealed myometrial invasion.

3. The total number of clinically diagnosed benign ovarian tumors was 29 , of which 2 cases had CA125 levels $>35 \mathrm{u} / \mathrm{ml}$ and on histopathology, they turned out to be malignant.

4. Clinically diagnosed malignant ovarian tumors were 37, in which 20 had raised levels of the biomarker. 


\section{ORIGINAL ARTICLE}

5. Of the 4 cases of adenomyosis, CA125 levels were elevated in 2 cases, and the histopathology of these cases revealed extensive adenomyosis. All the serous tumors showed significant elevation of CA125, one case of mucinous type had raised levels of the biomarker and histopathology revealed mucinous cyst adenoma.

6. The total number of metastatic carcinomas was 5 and CA125 levels in 4 of these were more than $35 \mathrm{u} / \mathrm{ml}$.

DISCUSSION: Various modalities have been adopted in the elusive diagnosis of the all pervasive pelvic mass. A biomarker that has been evaluated extensively is the CA125. In fact, it was proposed by Bast et al. as a relatively specific marker for ovarian malignancies. (3). Differentiating benign from early malignant ovarian disease is important and poses a diagnostic challenge. The combination of pelvic mass and elevated CA125 arouses suspicion of a gynecological malignancy but other conditions should always be considered in the differential diagnosis, especially in pre menopausal females. Malkaian et al studied 59 patients with histologically proven benign ovarian cysts, out of these 17 patients had elevated concentration of CA125 $(12>35 \mathrm{u} / \mathrm{ml}, 4>65 \mathrm{u} / \mathrm{ml}$ and $1>200 \mathrm{u} / \mathrm{ml})$. (7).

Chen DX et al studied 153 patients with benign pelvic masses and 58 with malignant pelvic masses and suggested serum CA 125 levels greater than $194 \mathrm{U} / \mathrm{ml}$ as a positivity criterion to differentiate benign and malignant ovarian tumors. (8). Nolen et al screened 85 biomarkers in patients with adnexal masses and more than half of the biomarkers differed significantly between benign and malignant masses.(9).

In our study CA125 levels were directly related to the size of the leiomyoma in concurrence with the study by P Bischof et al. (10).

Extensive adenomyosis was seen to be associated with raised levels of the biomarker in our study; Mitchel S Hoffman et al have reported similar outcomes in their study. (11).CA125 levels increased with the surgical staging of endometrial carcinoma and could be correlated with histopathology. These finding concurred with those of Nakayama et al, who also observed that increased CA125 levels indicated poor prognosis. (12).

The CA125 levels in benign serous epithelial germ cell and sex cord stromal tumor were less than $35 \mathrm{u} / \mathrm{ml}$. Mature ovarian teratoma and stroma ovarii (which belong to germ cell tumors of benign nature) in our study had CA125 levels $<35 \mathrm{u} / \mathrm{ml}$ ).

MS Tungurt et al have shown levels of CA125 to be in normal limits in mature cystic teratoma. (13). whereas Pei-fang Lai et al have shown elevated levels in malignant transformation of an ovarian mature cystic teratoma. (14).

Of the 23 malignant ovarian tumors 20 had CA125 levels $>35 \mathrm{U} / \mathrm{ml}$, the levels were high for all the malignant serous epithelial tumors and in the 2 cases that had raised levels histopathology revealed pseudomyxoma peritonei. Ramamani et al also in their study have shown CA125 to be a tumor marker for non-mucinous epithelial tumors. (15)

The CA125 levels were high in all endometrioid ovarian tumors, epithelial stromal tumors, epithelioid sarcoma and 4 of the 5 metastatic ovarian tumors.

CONCLUSION: The present study has highlighted the adjunct value of CA125 in myomas and extensive adenomyosis. It can be used pre operatively to suspect disease progression in 
endometrial carcinoma. The estimation of CA125can an additional tool to differentiate benign and malignant ovarian tumors. In postmenopausal age group, CA 125 levels were more than 35U/ml in all malignant ovarian tumors and less than $35 \mathrm{U} / \mathrm{ml}$ in all benign and borderline malignant ovarian tumors, thus sensitivity and specificity in this age group was 100\%.Clinical and histopathological findings in conjunction with CA125 could be adapted to advantage in the diagnosis of pelvic masses.

Table 1. Distribution of various associated conditions in the study group of patients with pelvic mass

\begin{tabular}{|l|l|l|}
\hline Associated Condition & Frequency & Percentage \\
\hline Hypertension & 16 & 20 \\
\hline Diabetes Mellitus & 14 & 17.5 \\
\hline Anemia & 13 & 16.25 \\
\hline Tuberculosis & 1 & 1.25 \\
\hline
\end{tabular}

Table 2.Frequency of different pelvic masses in the study group of patients

\begin{tabular}{|l|l|l|}
\hline Pelvic masses & No. of cases & Percentage \\
\hline Simple Ovarian Cyst & 8 & 10 \\
\hline Ovarian Tumour & 56 & 70 \\
\hline Fallopian Tube Carcinoma & 01 & 1,25 \\
\hline Leiomyoma & 06 & 7.5 \\
\hline Adenomyosis & 04 & 5 \\
\hline Pelvic Inflammatory disease presenting as mass & 01 & 1.25 \\
\hline Endometrial Carcinoma & 04 & 5 \\
\hline
\end{tabular}

Table 3. Different types of Ovarian tumours based on HIstopathological Report

\begin{tabular}{|l|l|l|l|l|}
\hline Ovarian Tumours & Benign & Malignant & Total & Percentage \\
\hline Surface epithelial & 27 & 19 & 46 & 82.1 \\
\hline Sex cord stromal & 01 & 00 & 01 & 1.8 \\
\hline Germ cell & 02 & 01 & 03 & 5.4 \\
\hline Sarcoma & 00 & 01 & 01 & 1.8 \\
\hline Metastatic & 00 & 05 & 05 & 8.9 \\
\hline Percentage & 62.5 & 37.5 & & 100 \\
\hline
\end{tabular}

Table 4 CA 125 levels in various pelvic masses in the study Group

\begin{tabular}{|l|l|l|l|l|}
\hline Pelvic mass & $<35 \mathrm{U} / \mathrm{ml}$ & $>35 \mathrm{U} / \mathrm{ml}$ & Total & Percentage \\
\hline Ovarian tumours & 35 & 21 & 56 & 70 \\
\hline Fibroid uterus & 03 & 03 & 06 & 7.5 \\
\hline Adenomyosis & 02 & 02 & 04 & 5 \\
\hline Endometrial carcinoma & 02 & 02 & 04 & 5 \\
\hline Fallopian tube carcinoma & 00 & 01 & 01 & 1,25 \\
\hline Chronic PID & 00 & 01 & 01 & 1,25 \\
\hline Ovarian cyst & 08 & 00 & 08 & 10 \\
\hline Percentage & 61,25 & 38.75 & & \\
\hline
\end{tabular}




\section{ORIGINAL ARTICLE}

\section{REFERENCES:}

1. Jung Woo Park,JeeHynn Park,Eun Seop Song Four Risk of Malignancy Indices (RMI) in evaluation of pelvic masses Korean J Obstet Gynaecol 2012;55(9):636-643

2. LeT,Giede C,Salem $S$ etal Initial evaluation and referral guidelines for management of pelvic/ovarian masses J Obstet Gynaecol Can 2009 Jul;31(7)648-80

3. Bast RC Jr, Feenay M, Lazarus H, Walden LM, Colven RB, Knapp RC: Reactivity of a monoclonal antibody with human ovarian carcinoma. J Clin Invest 1981; 68(5):1331-7.

4. Westhoff C. Ovarian cancer; Annu Rev Public Health 1996; 17:85-96.

5. Jacobs C, Bast RC Jr: The CA125 tumor associated antigen: a review of the literature Human Reprod 1989; 4(1):1-12.

6. Bast RC Jr, Klug TL, St. John E, Jensen and Niloff JM, Lazarus $\mathrm{H}$ et al. A radio immunoassay using monoclonal antibody to monitor the course of epithelial ovarian cancer. N Engl J Med 1983; 309(15):883-7.

7. Malkasian GD Jr, Knapp RC, Lavin PT, Zurawski VR Jr, Podratz KC et al; Preoperative evaluation of serum CA125 levels in premenopausal and postmenopausal patients with pelvic masses: differentiation of benign from malignant disease. Am J Obstet gynecol 1988 Aug;159(2):341-6

8. ChenDX, Schwartz PE, Li XG, Yang Z. Evaluation of CA125 levels in differentiating malignant from benign tumors in patients with pelvic masses. Obstet gynecol 1988; Jul 72:23-7.

9. Nolen BV, Elikokhatanaya L et al. Serum biomarker panels for the discrimination of benign from malignant cases in patients with an adnexal mass, Gynecol Oncol. 2010Jun; 117(3):440-5

10. PBishof, Galfetti MA et al Peripheral CA125 in patients with uterine fibroids. Hum Reprod.1992 Jan; 7(1):35-8.

11. Mitchel Hoffman, William N. Elevated rising CA125 with Adenomyosis Journal of Gynecologic SurgeryMarch.2001; Vol 17:33-34.

12. Nakayama Hiroki, Matsuschita Norhiro et al Do Serum CA125 levels predict poor prognosis in patients with endometrial cancer? Acta Obstetrica et Gynaecologica Japonica Vol.51;No12;1150-56.(1999)

13. Emin U.Tayfun G et al. Tumor Markers in mature cystic teratomas of the ovary. Archives of Gynecology and Obstetrics Feb2009, Vol 279(2):145-147.

14. Ramamani and Kaiser Jamil 2007 Specificity of serum tumor markers in ovarian malignancies. Trends in medical research 2:128-134. 